\title{
A RARE CASE OF SPONTANEOUS EXTERNAL ENDOMETRIOSIS ARISING FROM RECTUS ABDOMINIS MUSCLE
}

Radhika Rani Akkineni ${ }^{1}$, Ch. V. Pavan Kumar ${ }^{2}$

${ }^{1}$ Consultant, Department of Obstetrics and Gynaecology, Prime Hospital.

${ }^{2}$ Consultant, Department of General Surgery, Prime Hospital.

ABSTRACT

\section{BACKGROUND}

We report a case of 28 years old female P1L1 with 1 previous LSCS, presented with history of painful swelling in infraumbilical area about $5 \times 5 \mathrm{cms}$ below and right to the umbilicus, away from the caesarean scar. This case report aims to increase the awareness of this rare condition, which often misdiagnosed.

\section{KEYWORDS}

Endometriosis, Rectus Abdominis Muscle

HOW TO CITE THIS ARTICLE: Akkineni RR, Kumar CVP. A rare case of spontaneous external endometriosis arising from rectus abdominis muscle. J. Evolution Med. Dent. Sci. 2016;5(91):6806-6808, DOI: 10.14260/jemds/2016/1539

\section{BACKGROUND}

Endometriosis was first described by Rokitansky in 1860 and is defined as the presence and proliferation of functional endometrial tissue outside the uterine cavity, the commonest site being within the pelvis. Remote from the pelvic area, extrapelvic endometriosis has been documented in the vulva, urinary tract, gastrointestinal tract, chest wall, lungs and central nervous system.[1]

Abdominal wall endometriosis is very uncommon and is usually associated in the scar after previous pelvic surgery. We present the clinical findings, diagnostic features and treatment of a patient with abdominal endometriosis, which is a rare condition and often misdiagnosed.

\section{Case Report}

We report a case of 28 years old female P1L1 with 1 previous LSCS presented with history of painful swelling in infraumbilical area $\mathrm{x} 3$ months. The pain become worse during menstruation. She attained menarche at the age of 12 years. Her cycles were regular 28 - 30 days and menstrual period lasts for 4 - 5 days with normal flow. She had a delivery 5 years ago via caesarean section.

Examination findings were an ill-defined mass infraumbilically, $1 \mathrm{~cm}$ left to midline measuring $5 \times 5 \mathrm{cms}$, which was firm and tender. The tenderness persisted with contraction of her rectus abdominis muscle (Carnett's sign), implying extraperitoneal pathology. A Pfannenstiel incision was noted away from the mass. No lymph nodes were palpable. MRI of her abdomen showed lobulated multi-cystic lesion measuring $3.9 \times 3 \times 2.2 \mathrm{~cm}$ in size with small cystic component in left rectus abdominis muscle infraumbilical region features suggestive of endometriosis. FNAC of lesion showed clusters of degenerating cells, hemosiderin laden macrophages and polymorphs in a haemorrhagic background.

Financial or Other, Competing Interest: None.

Submission 20-09-2016, Peer Review 02-11-2016,

Acceptance 08-11-2016, Published 14-11-2016.

Corresponding Author:

Dr. Ch. V. Pavan Kumar,

Trendset Rhythm Apt, \#B-104,

White Field Area, Kondapur, Hyderabad.

E-mail: draradhikarani@gmail.com,chvpavan@yahoo.co.in

DOI: $10.14260 /$ jemds/2016/1539

\section{(c) (i) $\Theta$}

The mass explored under epidural anaesthesia via a transverse incision; $5 \times 4 \mathrm{~cm}$ mass was seen to be arising from anterior rectus sheath and rectus muscle. Wide local excision done and sent for histology. Defect in the muscle was repaired with overlay mesh.

The histology revealed muscle bundles and cystically dilated endometrial glands lined by pseudostratified columnar epithelium with extensive area of haemorrhage Figure 1 and 2. Postoperatively, the patient was started on norethisterone 5 mg TID for 90 days.

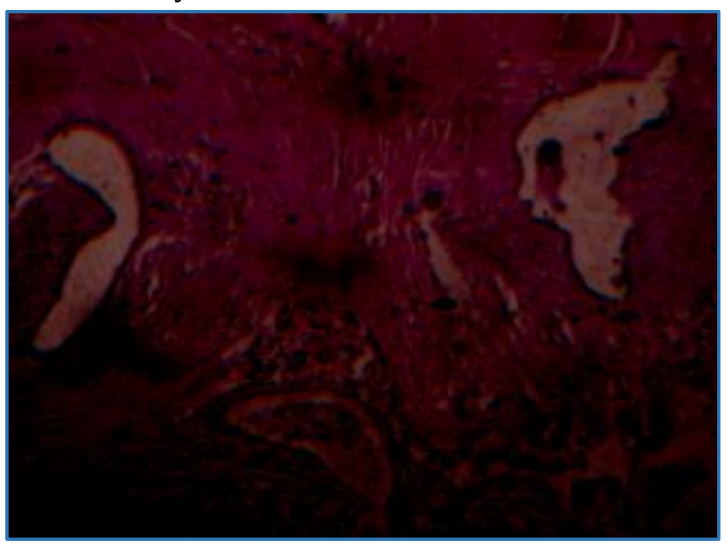

Figure 1 muscle bundles and cystically dilated endometrial glands lined by pseudostratified columnar epithelium with extensive area of haemorrhage

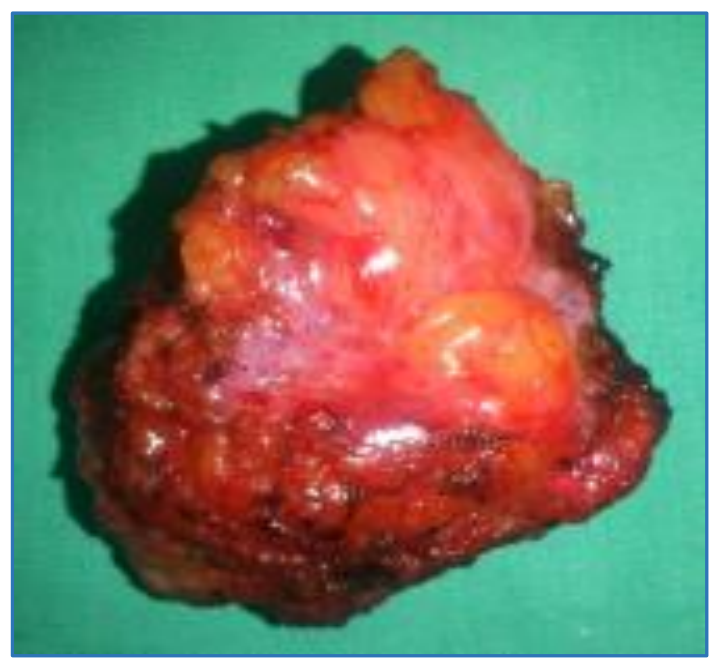

Figure 2.5 x $4 \mathrm{~cm}$ Mass Arising from Anterior Rectus Sheath and Muscle 


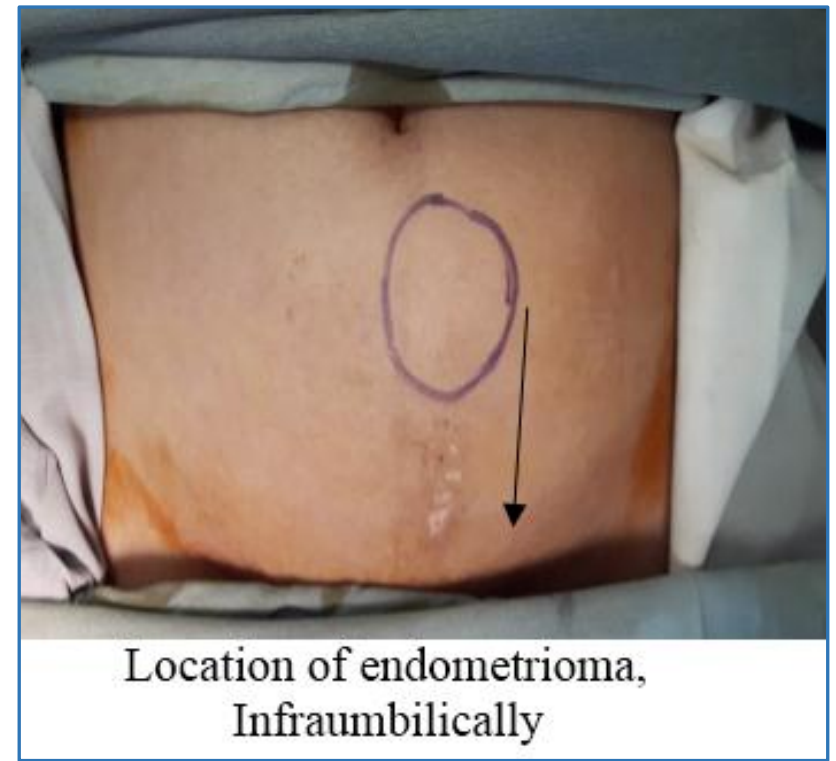

Figure 3

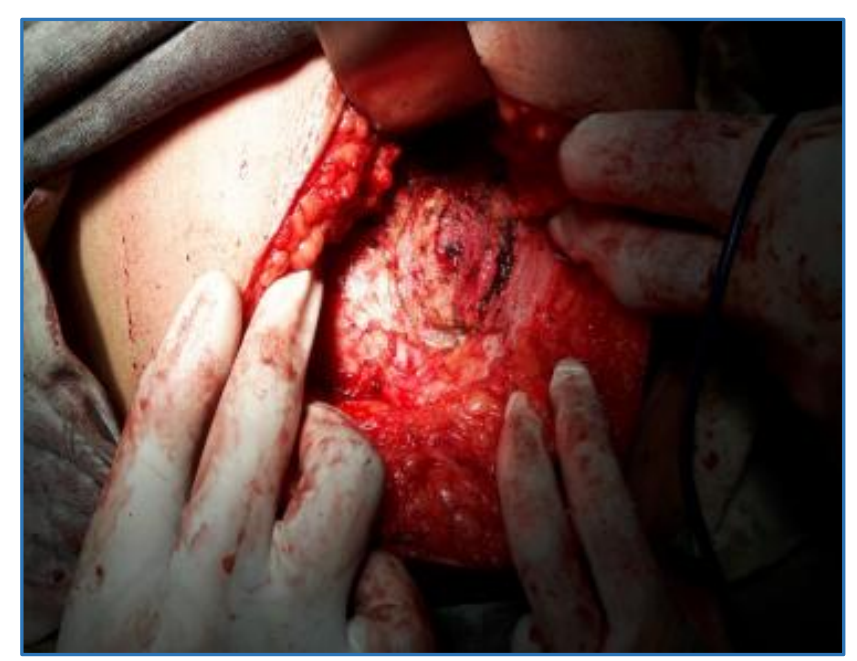

Figure 4. Endometrioma Arising from Rectus Sheath and Muscle

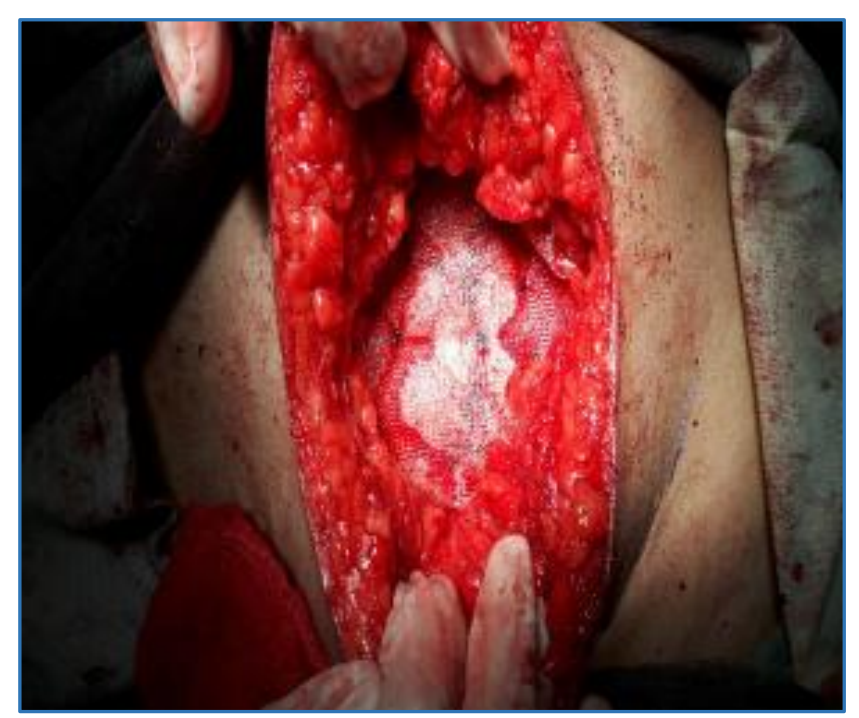

Figure 5. Repair of the Defect with Mesh

\section{DISCUSSION}

Endometriosis is defined as the presence of functional endometriotic tissue outside the uterine cavity.[2] Endometriosis most commonly affects the pelvic organs with the ovary being affected in $30-40 \%$ of cases and followed by fallopian tubes, bladder, rectum, vulva and pelvic ligaments.

External endometriosis is usually genitally located. Its extragenital locations are unusual and rare. The abdominal wall location is even rarer, representing 0.03 to $2 \%$ of all the extragenital parietal endometriosis. The abdominal parietal endometriosis has been described in different locations including caesarean scars, skin and surrounding tissues of abdominal or pelvic surgery scars, the site of passage of an amniocentesis needle or scars of laparoscopy. Abdominal wall endometriosis is very uncommon and is usually associated in the scar after previous surgery.

The ectopic endometrium responds to the cyclic hormonal changes and bleeding into surrounding stroma causing pain from pressure. The repeated episodes of bleeding gives rise to severe surrounding fibrosis and scarring. Our patient presented with severe pain over the lesion during periods.

The pathogenesis of caesarean scar endometriosis is best explained by many theories. The most popular theory is direct implantation; others are via lymphatic or haematogenous routes and subsequently grow into an endometrium. Abdominal wall endometrioma is generally seen at operation scar. In our case, endometrial tissue may have been sprinkled among the layers of the abdominal wall or may have been carried away from the operation site via the vascular pathway as a result of surgical trauma to the vessels during the operation.

The diagnosis of this location is often difficult. The cyclical nature of pain during menstruation often gives a clue towards diagnosis. The differential diagnosis of lump from abdominal wall are abscesses, haematoma, desmoid tumour, hernia, lipoma and rarely malignant tumours. Ultrasonography, MRI, FNAC are useful in diagnosis. FNAC can confirm the diagnosis by demonstrating epithelial cell, stromal cells and hemosiderin-laden macrophages.[3] MRI is gold standard, which can detect small lesions and confirm its vascularity.

Cancer antigen 125 (CA-125) is elevated in endometriosis and can be used to monitor treatment. It however is also elevated in gynaecological malignancies and in other conditions. Therefore, it is not diagnostic. [4]

Medical management of pelvic endometriosis include use of NSAID's, oral contraceptives, GnRH analogues. [5] Because of side effects and ineffectiveness of these treatments in cutaneous endometriosis, the treatment of choice in abdominal wall endometriosis is complete surgical excision including skin, subcutaneous tissue and rectus fascia with muscle. Excision of anterior abdominal wall may require mesh reconstruction. In our case prolene mesh was placed; 5 - 10 $\mathrm{mm}$ of surrounding healthy tissue to avoid re-implantation of microscopic remnants of endometrial tissue.

\section{CONCLUSION}

Endometriosis arising from rectus muscle is a rare condition and often misdiagnosed. In female patients with mass in the lower abdominal wall with symptoms in correlation with menstruation, especially after caesarean section, endometrioma as well as desmoid tumour should be 
suspected. CT and MRI support the diagnosis. FNAC can be used to obtain a cellular diagnosis. Wide local excision of lesion is the treatment of choice.

\section{REFERENCES}

1. Erkan N, Haciyanli M, Sayhan H. Abdominal wall endometriosis. Int J Gynaecol Obstet 2005;89(1):59-60.

2. Yi TM, Kim YJ, Kim SG, et al. A case of endometrioma at the abdominal scar. Journal of the Korean Society of Plastic and Reconstructive Surgeons 2010;37(6):843-6.
3. Veda $P$, Srinivasaiah $M$. Incisional endometriosis: diagnosed by fine needle aspiration cytology. J Lab Physicians 2010;2(2):117-20.

4. Szubert M, Suzin J, Wierzbowski T, et al. CA-125 concentration in serum and peritoneal fluid in patients with endometriosis - preliminary results. Arch Med Sci 2012;8(3):504-8.

5. Prentice A. Endometriosis. British Medical Journal 2001;323(7304):93-5. 